\title{
EFFECTS OF INERTIA IN THE STEADY STATE PRESSURISED FLOW OF A NON-NEWTONIAN FLUID BETWEEN TWO CURVILINEAR SURFACES OF REVOLUTION: RABINOWITSCH FLUID MODEL
}

\author{
Udaya P. Singh $^{* 1}$, Ram S. Gupta ${ }^{2}$, Vijay K. Kapur ${ }^{2}$ \\ ${ }^{1}$ Ambalika Institute of Management \& Technology, Mohanlal Ganj, Lucknow, U.P., India \\ ${ }^{2}$ Kamla Nehru Institute of Technology, Sultanpur, U.P., India
}

\begin{abstract}
In many practical situations fluids are normally blended with additives (viscosity index improvers, viscosity thickeners, viscosity thinners) due to which they show pseudoplastic and dilatant nature which can be modelled as cubic stress model (Rabinowitsch model). The cubic stress model for pseudoplastic fluids is adopted because Wada and Hayashi have shown that the theoretical results with this model are in good agreement with the experimental results. The present theoretical analysis is to investigate the pseudoplastic effect along with the effect of rotational inertia on the pressure distribution, frictional torque and fluid flow rate of externally pressurised flow in narrow clearance between two curvilinear surfaces of revolution. The expression for pressure has been derived using energy integral approach. To analyse and discuss the effects of pseudoplasticity and fluid inertia on the pressure distribution, fluid flow rate and frictional torque, the examples of externally pressurised flow in the clearance between parallel disks and concentric spherical surfaces have been considered.
\end{abstract}

Keywords: curvilinear bearings, externally pressurized flow, Rabinowitsch fluid model, inertia effect

\section{INTRODUCTION}

In many practical lubrication applications, the classical Newtonian theory is not a satisfactory engineering approach. Experiments show that the addition of small amounts of long-chained polymer additives to a Newtonian fluid can produce desirable lubricants. Such lubricants behave as nonNewtonian pseudoplastic, dilatant and viscoelastic fluids according to the nature and quantity of additives. Dilatant fluids are not of much importance in practical use but the effect of pseudoplasticity on lubrication characteristics has recently taken on added significance with the move to yet lowerviscosity lubricants for improved energy efficiency. In recent years, tribologists have done a great deal of work to increase the efficiency of stabilising properties of non-Newtonian lubricants by addition of small amounts of long chain polymer solutions such as polyisobutylene and ethylene propylene etc. The use of additives minimises the sensitivity of the lubricant to changes in the shearing strain rate. Further, the viscosity of these lubricants exhibits a non-linear relationship between the shearing stress and shearing strain rate. In last few decades, the rheological effects of non-Newtonian lubricants based on different fluid models such as power law and couple stress fluid model have been studied for the performance characteristics of journal, squeeze film, annular disks and externally pressurised bearings. The theoretical investigation of the problems of bearing lubrication and its performance using NonNewtonian lubricants was done by Giannikos and Buckholz (1988) for elastic bearings with nonNewtonian power law fluids, (Khonsari and Brewe, 1989) for journal bearing with micropolar fluids, 
Serangi et al. (2005) for ball bearing with couple stress fluid. The analysis of bearings' performance with pseudoplastic fluids was done by Savins (1958) for flow in stationary pipes, (Cross, 1965) generating a new flow equation, (Hanks, 1979) for flow in concentric annuls, (Hasmimoto and Wada, 1986; Shukla and Prasad, 1982) on squeeze films, (Usha and Vimla, 2000) on squeeze film bearings and the analysis of externally pressurised bearings was done by (Lin, 1999) on plane stepped plate bearing with couple stress model, (Jurczak et al., 2006; Walicka and Falicki, 2010 $2010^{\mathrm{b}}$ ) on flow in the clearance between the two curvilinear surfaces of revolution with Casson and Herschel-Bulkley and couple stress fluid models. But the problem of pressurised flow in the clearance between the two curvilinear surfaces of revolution considering the inertia effect using pseudoplastic fluid - Rabinowitsch fluid model to establish the non-linear relationship between the shearing stress and shearing strain rate is yet to be considered.

In the Rabinowitsch fluid model, the following empirical relation holds for one dimensional fluid flow:

$$
\tau_{x z}+\kappa \tau_{x z}^{3}=\mu \frac{\partial u}{\partial z}
$$

where $\mu$ is the zero shear rate viscosity, $\kappa$ is the non-linear factor responsible for the non-Newtonian effects of the fluid which will be referred to as coefficient of pseudoplasticity in this paper. This model can be applied to Newtonian lubricants for $\kappa=0$, to dilatant lubricants for $\kappa<0$, and to pseudoplastic lubricants for $\kappa>0$. The experimental analysis of this model for the lubricants for journal bearing has been justified by (Wada and Hayashi, 1971) indicating the film pressure and load capacity for these lubricants is smaller than those of the Newtonian fluids. Afterwards, the theoretical study of bearing performance with non-Newtonian lubricants using this model was done by (Bourging and Gay, 1984) on Journal bearing, (Hsu and Saibel, 1965; Lin, 2001) on slider bearing, (Hashmimoto and Wada, 1986) on circular plates bearing and Hung (2009) on infinitely wide parallel rectangular plates. However, none of the investigators has focused their attention to study theoretically, the problem of isothermal, incompressible laminar flow lubricant for externally pressurised bearings taking into account the Rabinowitsch fluid model.

In the present paper, effects of pseudoplasticity and inertia in the pressurised flow of a non-Newtonian fluid between two curvilinear surfaces of revolution have been investigated using Rabinowitsch fluid model. Since the problem is of non-linear nature in its theoretical investigation, the energy integral approach presented by (Elkouh, 1967) and established by (Kapur and Verma, 1973) is used to calculate pressure, load capacity and frictional torque.

\section{CONSTITUTIVE EQUATIONS AND BOUNDARY CONDITIONS}

The physical configuration of externally pressurised flow in the clearance between two curvilinear surfaces of revolution is shown in Fig. 1. The radius of revolution of the outer surface is $r(x)$ and the clearance between the two surfaces of revolution is $h(x)$. In Fig. 1, an intrinsic curvilinear orthogonal coordinate system $x, \vartheta, z$ is also shown. The lubricant in the system is taken as non-Newtonian. The body forces and body couples are assumed to be absent. Under the assumptions of hydrodynamic lubrication applicable to thin film as considered by (Cameron, 1996; Pinkus \& Sternlitch, 1961) and the assumptions of thin films in curvilinear coordinate used and discussed by (Jurczak et al., 2006), the field equations governing the one dimensional motion of an incompressible non-Newtonian fluid Rabinowitsch fluid model in curvilinear co-ordinate system are:

$$
\frac{1}{r(x)} \frac{\partial}{\partial x}[r(x) u]=0
$$



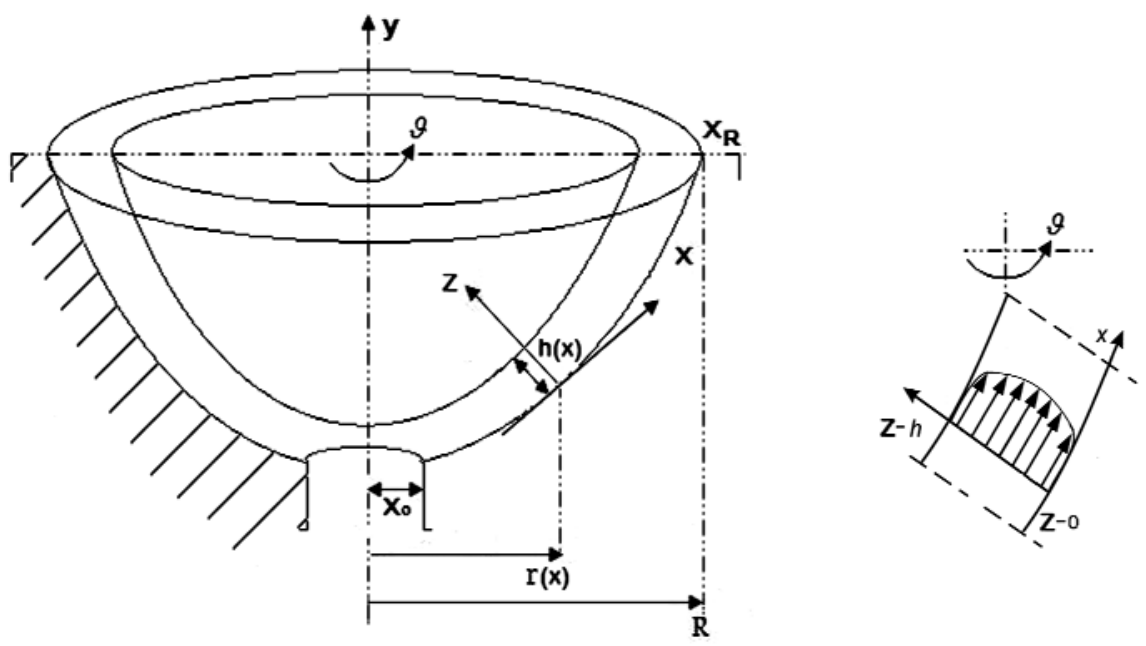

Fig. 1. Schematic diagram of curvilinear clearance between two surfaces of revolution

$$
\begin{gathered}
\frac{\partial \tau_{z x}}{\partial z}=\frac{\partial p}{\partial x}-\frac{\rho v^{2}}{r(x)} r^{\prime}(x) \\
\mu \frac{\partial^{2} v}{\partial z^{2}}=0 \\
\frac{\partial p}{\partial z}=0
\end{gathered}
$$

where $r^{\prime}(\mathrm{x})$ is the derivative of $r(\mathrm{x})$ with respect to $\mathrm{x}$.

Equations (2) - (4) are solved under the following boundary conditions:

$$
\begin{array}{ll}
u=0 & \text { at } z=0, h \\
v=0 & \text { at } z=0 \\
v=r(x) \omega & \text { at } z=h \\
p=p_{0} & \text { at } x=x_{0} \\
p=0 & \text { at } x=x_{R}
\end{array}
$$

where $u \& v$ are the velocity components of flow as shown in Figure 1, $\omega$ is the angular velocity of inner surface, $\rho$ is density of fluid, $p$ is film pressure between bearing surfaces and $\tau_{x z}$ is shearing stress.

Introducing the dimensionless quantities

$$
\begin{gathered}
\tilde{x}=\frac{x}{x_{R}}, \quad \tilde{z}=\frac{z}{x_{R}}, \quad \tilde{h}=\frac{h}{x_{R}}, \quad \tilde{x}_{O}=\frac{x_{O}}{x_{R}}, \quad \tilde{r}(\tilde{x})=\frac{r(x)}{R}, \\
\tilde{p}=\frac{p}{p_{O}} \quad \tilde{u}=\frac{u}{R \omega} \quad \tilde{v}=\frac{v}{R \omega} \quad \tilde{\tau}_{z x}=\frac{\tau_{z x}}{p_{O}} \quad \alpha=\kappa p_{O}^{2} \\
S=\frac{3}{20} \frac{\rho R^{2} \omega^{2}}{p_{O}}, \quad \delta=\frac{\mu_{N} R \omega}{p_{O} x_{R}}, \quad \tilde{\mu}=\frac{\mu}{\mu_{N}}
\end{gathered}
$$

the field equations, Equation (1) to Equation (5), are transformed to

$$
\frac{1}{\tilde{r}(\tilde{x})} \frac{\partial}{\partial \tilde{x}}(\tilde{r}(\tilde{x}) \tilde{u})=0
$$




$$
\begin{gathered}
\frac{\partial \tilde{\tau}_{z x}}{\partial \tilde{z}}=\frac{\partial \tilde{p}}{\partial \tilde{x}}-\frac{20}{3} s \frac{\tilde{v}^{2}}{\tilde{r}(\tilde{x})} \tilde{r}^{\prime}(\tilde{x}) \\
\tilde{\mu} \frac{\partial^{2} \tilde{v}}{\partial \tilde{z}^{2}}=0 \\
\frac{\partial \tilde{P}}{\partial \tilde{z}}=0 \\
\tilde{\tau}_{z x}+\alpha \tilde{\tau}_{z x}^{3}=\delta \tilde{\mu} \frac{\partial \tilde{u}}{\partial \tilde{z}}
\end{gathered}
$$

and the related boundary conditions (6) to (8) take the form:

$$
\begin{array}{crc}
\tilde{u}=0 & \text { at } & \tilde{z}=0, \tilde{h} \\
\tilde{v}=0 & \text { at } & \tilde{z}=0 \\
\tilde{v}=\tilde{r}(\tilde{x}) & \text { at } & \tilde{z}=\tilde{h} \\
\tilde{p}=1 & \text { at } & \tilde{x}=\tilde{x}_{o} \\
\tilde{p}=0 & \text { at } & \tilde{x}=1
\end{array}
$$

\subsection{Analysis}

The solution for Equation (12) satisfying Equation (11) and the boundary conditions (16) ignoring the inertia effects is

$$
\tilde{u}=\frac{1}{2 \delta \tilde{\mu}}\left[\frac{\partial \tilde{p}}{\partial \tilde{x}}\left(\tilde{z}^{2}-\tilde{z} \tilde{h}\right)+\alpha\left(\frac{\partial \tilde{p}}{\partial \tilde{x}}\right)^{3}\left(\frac{\tilde{z}^{4}}{2}-\tilde{z}^{3} \tilde{h}+\frac{3}{4} \tilde{z}^{2} \tilde{h}^{2}-\frac{1}{4} \tilde{z} \tilde{h}^{3}\right)\right]
$$

From the continuity of flow equation (11), the rate of change of film pressure $\frac{\partial \tilde{p}}{\partial \tilde{x}}$ without inertia effect can be approximated, in terms of radial flow rate $\tilde{Q}$, as

$$
\frac{\partial \tilde{p}}{\partial \tilde{x}}=-\frac{6 \tilde{\mu} \tilde{Q}}{\tilde{r}(\tilde{x}) \tilde{h}^{3}}+\frac{162}{5} \alpha \frac{\tilde{\mu}^{3} \tilde{Q}^{3}}{\tilde{r}(\tilde{x})^{3} \tilde{h}^{7}}
$$

where $\widetilde{Q}=\frac{\mu_{N}}{\pi R x_{R}^{2} p_{O}} Q$, and $Q=2 \pi r(x) \int_{0}^{h(x)} u \mathrm{~d} z$

Substituting this expression for $\frac{\partial \tilde{p}}{\partial \tilde{x}}$ from Equation (22) in Equation (21), velocity profile for $\tilde{u}$ without the inertia effect is as

$$
\tilde{u}=\frac{1}{2 \delta \tilde{\mu}}\left[f(\tilde{x})\left(\tilde{z}^{2}-\tilde{z} \tilde{h}\right)+\alpha\{f(\tilde{x})\}^{3}\left(\frac{\tilde{z}^{4}}{2}-\tilde{z}^{3} \tilde{h}+\frac{3}{4} \tilde{z}^{2} \tilde{h}^{2}-\frac{1}{4} \tilde{z} \tilde{h}^{3}\right)\right]
$$

where 


$$
f(\tilde{x})=-\frac{6 \tilde{\mu} \tilde{Q}}{\tilde{r}(\tilde{x}) \tilde{h}^{3}}+\frac{162}{5} \alpha \frac{\tilde{\mu}^{3} \tilde{Q}^{3}}{\tilde{r}(\tilde{x})^{3} \tilde{h}^{7}}
$$

The circumferential velocity $v$ can be found by integrating Equation (13) under the boundary conditions (17) and (18) as

$$
\tilde{v}=\tilde{r} \frac{\tilde{z}}{\tilde{h}}
$$

Multiply Equation (12) by $\tilde{r}(\tilde{x}) \tilde{u}$ and integrating the resulting expression from $\tilde{z}=0$ to $\tilde{z}=\tilde{h}$, the following expression is obtained using Energy Integral Method (Elkouh, 1967; Kapur and Verma, 1973)

$$
2 \delta \tilde{r}(\tilde{x}) \int_{0}^{\tilde{h}}\left(\tilde{u} \frac{\partial \tilde{\tau}_{z x}}{\partial \tilde{z}}\right) \mathrm{d} \tilde{z}=\tilde{Q} \frac{\partial \tilde{p}}{\partial \tilde{x}}-\frac{40 S \delta}{3} \int_{0}^{\tilde{h}}\left\{\tilde{u} \tilde{v}^{2} \tilde{r}^{\prime}(\tilde{x})\right\} \mathrm{d} \tilde{z}
$$

Using Equations (23) - (26) the rate of change of modified pressure in $x$ direction with inertia effect is obtained as:

$$
\frac{\mathrm{d} \tilde{\mathrm{p}}}{\mathrm{d} \tilde{x}}=\frac{1}{\tilde{\mu} \tilde{Q}}\left[-\frac{1}{6} \tilde{r}(\tilde{x}) \tilde{h}^{3} f(\tilde{x})^{2}-\frac{\alpha}{40} \tilde{r}(\tilde{x}) \tilde{h}^{5} f(\tilde{x})^{4}-\frac{20 S}{3} \tilde{r}^{\prime}(\tilde{x})\left\{\frac{1}{20} \tilde{r}(\tilde{x})^{2} \tilde{h}^{3} f(\tilde{x})+\frac{13 \alpha}{1680} \tilde{r}(\tilde{x})^{2} \tilde{h}^{5} f(\tilde{x})^{3}\right\}\right]
$$

\section{EXAMPLES OF APPLICATIONS}

For the presentation of work done here, two examples of parallel plates bearings and concentric spherical bearings are taken into consideration.

\subsection{Plane parallel plates}

Taking the following transformations of the parameters

$$
\begin{array}{ll}
x=r(x)=r, & x_{R}=R, \quad x_{O}=r_{O}, \quad h=\text { constant clearance, } \\
\tilde{x}_{O}=\tilde{r}_{O}, \quad \tilde{x}=\tilde{r}(\tilde{x})=\tilde{r}, \quad \tilde{r}^{\prime}(\tilde{x})=1, &
\end{array}
$$

the curvilinear bearing (as shown in Fig. 1.) can be considered as the plane parallel plates shown in Fig. 2 .

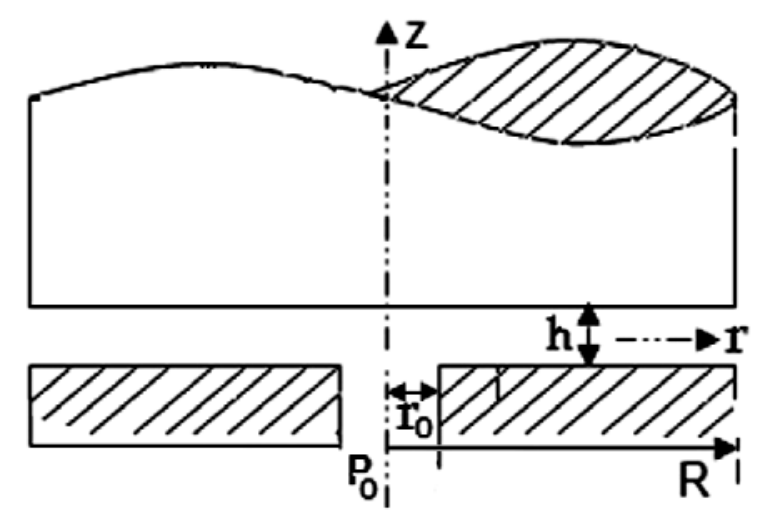

Fig. 2. Schematic diagram clearance between two parallel disks 
With these simplifications, Equation (27) becomes

$$
\frac{\mathrm{d} \tilde{\mathrm{p}}}{\mathrm{d} \tilde{r}}=\frac{1}{\tilde{\mu} \tilde{Q}}\left[-\frac{1}{6} \tilde{r} \tilde{h}^{3} f(\tilde{r})^{2}-\frac{\alpha}{40} \tilde{r} \tilde{h}^{5} f(\tilde{r})^{4}-\frac{20 S}{3}\left\{\frac{1}{20} \tilde{r}^{2} \tilde{h}^{3} f(\tilde{r})+\frac{13 \alpha}{1680} \tilde{r}^{2} \tilde{h}^{5} f(\tilde{r})^{3}\right\}\right]
$$

Integrating Equation (28) under boundary conditions

$$
\begin{array}{lll}
\tilde{p}=1 & \text { at } & \tilde{r}=\tilde{r}_{O} \\
\tilde{p}=0 & \text { at } & \tilde{r}=1
\end{array}
$$

the expression for pressure distribution is obtained as

$$
\begin{aligned}
\tilde{p}(\tilde{r})=\tilde{r}^{2} \tilde{s} & -\frac{81 \tilde{Q}^{3} \alpha \tilde{\mu}^{3}}{5 \tilde{h}^{7} \tilde{r}^{2}}+\frac{3159 \tilde{Q}^{4} s \alpha^{2} \tilde{\mu}^{4}}{35 \tilde{h}^{8} \tilde{r}^{2}}-\frac{6561 \tilde{Q}^{5} \alpha^{2} \tilde{\mu}^{5}}{50 \tilde{h}^{11} \tilde{r}^{4}}-\frac{85293 \tilde{Q}^{6} s \alpha^{3} \tilde{\mu}^{6}}{350 \tilde{h}^{12} \tilde{r}^{4}}+\frac{118098 \tilde{Q}^{7} \alpha^{3} \tilde{\mu}^{7}}{125 \tilde{h}^{15} \tilde{r}^{6}} \\
& +\frac{255879 \tilde{Q}^{8} s \alpha^{4} \tilde{\mu}^{8}}{875 \tilde{h}^{16} \tilde{r}^{6}}-\frac{1594323 \tilde{Q}^{9} \alpha^{4} \tilde{\mu}^{9}}{625 \tilde{h}^{19} \tilde{r}^{8}}+\frac{43046721 \tilde{Q}^{11} \alpha^{5} \tilde{\mu}^{11}}{15625 \tilde{h}^{23} \tilde{r}^{10}}-\frac{6 \tilde{Q} \tilde{\mu} \log [\tilde{r}]}{\tilde{h}^{3}} \\
& +\frac{12 \tilde{Q}^{2} s \alpha \tilde{\mu}^{2} \log [\tilde{r}]}{35 h^{4}}-S+\frac{81 \tilde{Q}^{3} \alpha \tilde{\mu}^{3}}{5 \tilde{h}^{7}}-\frac{3159 \tilde{Q}^{4} s \alpha^{2} \tilde{\mu}^{4}}{35 \tilde{h}^{8}}+\frac{6561 \tilde{Q}^{5} \alpha^{2} \tilde{\mu}^{5}}{50 \tilde{h}^{11}}+\frac{85293 \tilde{Q}^{6} s \alpha^{3} \tilde{\mu}^{6}}{350 \tilde{h}^{12}} \\
& -\frac{118098 \tilde{Q}^{7} \alpha^{3} \tilde{\mu}^{7}}{125 \tilde{h}^{15}}-\frac{255879 \tilde{Q}^{8} s \alpha^{4} \tilde{\mu}^{8}}{875 \tilde{h}^{16}}+\frac{1594323 \tilde{Q}^{9} \alpha^{4} \tilde{\mu}^{9}}{625 \tilde{h}^{19}}-\frac{43046721 \tilde{Q}^{11} \alpha^{5} \tilde{\mu}^{11}}{15625 \tilde{h}^{23}}
\end{aligned}
$$

for $\tilde{r}_{O} \leq \tilde{r} \leq 1$

\subsection{Concentric spherical surfaces}

Taking the following transformations of the parameters

$\tilde{x}=\frac{x}{x_{R}}=\frac{R \theta}{R \frac{\pi}{2}}=\frac{2 \theta}{\pi}, \quad \tilde{r}(\tilde{x})=\frac{r(x)}{R}=\operatorname{Sin}(\theta)$,

$\frac{\mathrm{d} \tilde{r}(\tilde{x})}{\mathrm{d} \tilde{x}}=\frac{\pi}{2} \operatorname{Cos}(\theta), \quad \frac{\mathrm{d} \tilde{p}}{\mathrm{~d} \tilde{x}}=\frac{\pi}{2} \frac{\mathrm{d} \tilde{p}}{\mathrm{~d} \theta}$ and $\tilde{h}=$ constant clearance,

the curvilinear bearing (as shown in Fig. 1.) can be considered as the concentric spherical surfaces shown in Fig. 3.

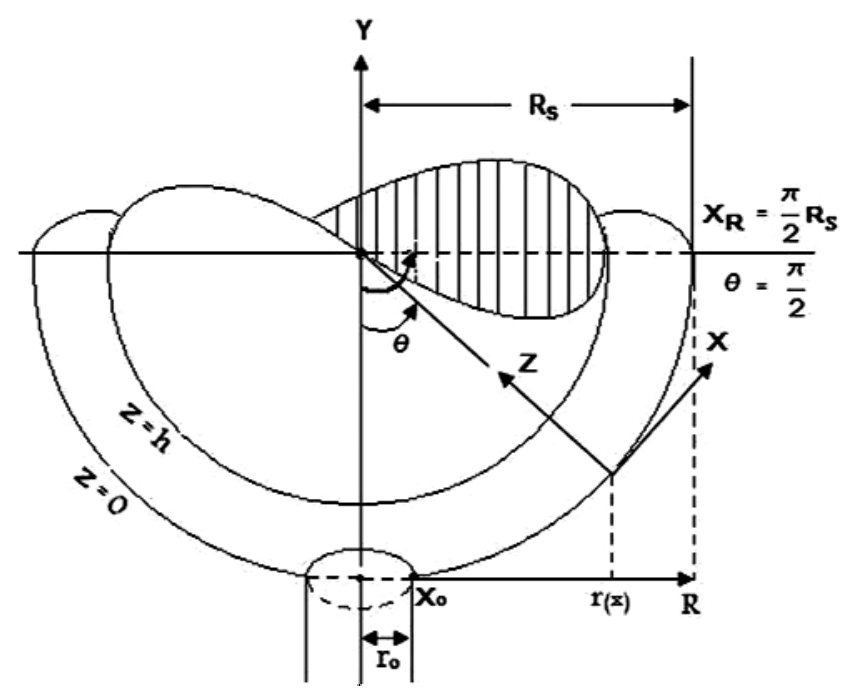

Fig. 3. Schematic diagram of clearance between two concentric spherical surfaces 
In this case, Equation (27) becomes

$$
\begin{aligned}
\frac{\mathrm{d} \tilde{\mathrm{p}}(\theta)}{\mathrm{d} \theta}= & \frac{-1}{6 \tilde{\mu} \tilde{Q}}\left[S \frac{\pi}{2} \operatorname{Cos}(\theta)\left(2 \operatorname{Sin}(\theta)^{2} \tilde{h}^{3} f(\theta)+\frac{13}{42} \alpha \operatorname{Sin}(\theta)^{2} \tilde{h}^{5} f(\theta)^{3}\right)\right. \\
& +\operatorname{Sin}(\theta) \tilde{h}^{3} f(\theta)^{2}+\frac{3}{20} \alpha \operatorname{Sin}(\theta) \tilde{h}^{5} f(\theta)
\end{aligned}
$$

where

$$
f(\theta)=-6 \frac{\tilde{\mu} \tilde{Q}}{\operatorname{Sin}(\theta) \tilde{h}^{3}}+\frac{162}{5} \alpha \frac{\tilde{\mu}^{3} \tilde{Q}^{3}}{\operatorname{Sin}(\theta)^{3} \tilde{h}^{7}}
$$

Integrating Equation (30) under boundary conditions

$$
\begin{array}{lll}
\tilde{p}=1 & \text { at } & \theta=\theta_{O} \\
\tilde{p}=0 & \text { at } & \theta=\pi / 2
\end{array}
$$

the expression for pressure distribution, leaving the terms that have order less than $10^{-8}$, is obtained as

$$
\begin{aligned}
\tilde{p}(\theta)= & \frac{1}{175 \tilde{h}^{12} \pi}\left[-\frac{175}{2} \tilde{h}^{12} \pi \operatorname{Cos}(2 \theta)-\frac{567}{8}\left(20 \tilde{h}^{5} \tilde{Q}^{3} \alpha \tilde{\mu}^{3}+243 \tilde{h} \tilde{Q}^{5} \alpha^{2} \tilde{\mu}^{5}\right)\right. \\
& \times \operatorname{Csc}(\theta / 2)^{2}-\frac{45927 \tilde{h} \tilde{Q}^{5} \alpha^{2} \tilde{\mu}^{5}}{16 \operatorname{Sin}(\theta / 2)^{4}+15795 \tilde{h}^{4} \pi \tilde{Q}^{4} \alpha^{2} \tilde{\mu}^{4} \operatorname{Csc}(\theta)^{2}} \\
& -\frac{85293}{2} \pi \tilde{Q}^{6} \operatorname{s\alpha }^{3} \tilde{\mu}^{6} \operatorname{Csc}(\theta)^{4}+\frac{21}{2}\left(200 \tilde{h}^{9} \tilde{Q} \tilde{\mu}-540 \tilde{h}^{5} \tilde{Q}^{3} \alpha \tilde{\mu}^{3}\right. \\
& \left.-6561 \tilde{h} \tilde{Q}^{5} \alpha^{2} \tilde{\mu}^{5}\right) \log (\operatorname{Cos}(\theta / 2))-\frac{21}{2}\left(200 \tilde{h}^{9} \tilde{Q} \tilde{\mu}-540 \tilde{h}^{5} \tilde{Q}^{3} \alpha \tilde{\mu}^{3}\right. \\
& \left.-6561 \tilde{h} \tilde{Q}^{5} \alpha^{2} \tilde{\mu}^{5}\right) \log (\operatorname{Sin}(\theta / 2))+60 \tilde{h}^{8} \pi \tilde{Q}^{2} \operatorname{so} \tilde{\mu}^{2} \log (\operatorname{Sin}(\theta)) \\
& +\frac{567\left(20 \tilde{h}^{5} \tilde{Q}^{3} \alpha \tilde{\mu}^{3}+243 \tilde{h} \tilde{Q}^{5} \alpha^{2} \tilde{\mu}^{5}\right)}{\left.8 \operatorname{Cos}(\theta / 2)^{2}+\frac{45927}{16} \tilde{h} \tilde{Q}^{5} \alpha^{2} \tilde{\mu}^{5} \operatorname{Sec}(\theta / 2)^{4}\right]} \\
& -\frac{\left(31590 \tilde{h}^{4} \pi \tilde{Q}^{4} \alpha^{2} \tilde{\mu}^{4}-85293 \pi \tilde{Q}^{6} \alpha^{3} \tilde{\mu}^{6}\right)}{350 \tilde{h}^{12} \pi}-\frac{s}{2}
\end{aligned}
$$

for $\theta_{o} \leq \theta \leq \pi / 2$

\section{LUBRICANT FLOW RATE}

\subsection{Plane parallel plates}

From the equation of pressure distribution Equation (29) and the related boundary conditions, the nonlinear expression for radial flow rate $(\tilde{Q})$ is obtained as 


$$
\begin{aligned}
& 1+s-\tilde{r}_{o}^{2} s-\frac{81 \tilde{Q}^{3} \alpha \tilde{\mu}^{3}}{5 \tilde{h}^{7}}+\frac{81 \tilde{Q}^{3} \alpha \tilde{\mu}^{3}}{5 \tilde{h}^{7} \tilde{r}_{o}^{2}}+\frac{3159 \tilde{Q}^{4} s \alpha^{2} \tilde{\mu}^{4}}{35 \tilde{h}^{8}}-\frac{3159 \tilde{Q}^{4} s \alpha^{2} \tilde{\mu}^{4}}{35 \tilde{h}^{8} \tilde{r}_{o}^{2}} \\
& -\frac{6561 \tilde{Q}^{5} \alpha^{2} \tilde{\mu}^{5}}{50 \tilde{h}^{11}}+\frac{6561 \tilde{Q}^{5} \alpha^{2} \tilde{\mu}^{5}}{50 \tilde{h}^{11} \tilde{r}_{o}}-\frac{85293 \tilde{Q}^{6} s \alpha^{3} \tilde{\mu}^{6}}{350 \tilde{h}^{12}}+\frac{85293 \tilde{Q}^{6} s \alpha^{3} \tilde{\mu}^{6}}{350 \tilde{h}^{12} \tilde{r}_{o}^{4}} \\
& +\frac{6 \tilde{Q} \tilde{\mu} \log \left[\tilde{r}_{o}\right]}{\tilde{h}^{3}}-\frac{12 \tilde{Q}^{2} s \alpha \tilde{\mu}^{2} \log \left[\tilde{r}_{o}\right]}{35 \tilde{h}^{4}}=0=F(\tilde{Q})
\end{aligned}
$$

\subsection{Concentric spherical surfaces}

From the equation of pressure distribution equation (32) and the related boundary conditions, the nonlinear expression for flow rate $(\tilde{Q})$ is obtained as

$$
\begin{aligned}
& 1+\frac{s}{2}+\frac{3159 \tilde{Q}^{4} s \alpha^{2} \tilde{\mu}^{4}}{35 \tilde{h}^{8}}-\frac{85293 \tilde{Q}^{6} s \alpha^{3} \tilde{\mu}^{6}}{350 \tilde{h}^{12}}+\frac{1}{2} \operatorname{sCos}(2 \theta o)+\frac{81 \tilde{Q}^{3} \alpha \tilde{\mu}^{3} \operatorname{Csc}(\theta o / 2)^{2}}{10 \tilde{h}^{7} \pi} \\
& +\frac{19683 \tilde{Q}^{5} \alpha^{2} \tilde{\mu}^{5} \operatorname{Csc}(\theta o / 2)^{2}}{200 \tilde{h}^{11} \pi}+\frac{6561 \tilde{Q}^{5} \alpha^{2} \tilde{\mu}^{5} \operatorname{Csc}(\theta o / 2)^{4}}{400 \tilde{h}^{11} \pi}-\frac{3159 \tilde{Q}^{4} \operatorname{s} \alpha^{2} \tilde{\mu}^{4} \operatorname{Csc}(\theta o)^{2}}{35 \tilde{h}^{8}} \\
& +\frac{85293 \tilde{Q}^{6} \operatorname{s} \alpha^{3} \tilde{\mu}^{6} \operatorname{Csc}(\theta o)^{4}}{350 \tilde{h}^{12}}-\frac{12 \tilde{Q} \tilde{\mu} \log (\operatorname{Cos} \theta o / 2)}{\tilde{h}^{3} \pi}+\frac{162 \tilde{Q}^{3} \alpha \tilde{\mu}^{3} \log (\operatorname{Cos}(\theta o / 2))}{5 \tilde{h}^{7} \pi} \\
& +\frac{19683 \tilde{Q}^{5} \alpha^{2} \tilde{\mu}^{5} \log (\operatorname{Cos}(\theta o / 2))}{50 \tilde{h}^{11} \pi}+\frac{12 \tilde{Q} \tilde{\mu} \log (\operatorname{Sin}(\theta o / 2))}{\tilde{h}^{3} \pi}-\frac{162 \tilde{Q}^{3} \alpha \tilde{\mu}^{3} \log (\operatorname{Sin}(\theta o / 2))}{5 \tilde{h}^{7} \pi} \\
& -\frac{19683 \tilde{Q}^{5} \alpha^{2} \tilde{\mu}^{5} \log (\operatorname{Sin}(\theta o / 2))}{50 \tilde{h}^{11} \pi}-\frac{12 \tilde{Q}^{2} \operatorname{s\alpha } \tilde{\mu}^{2} \log (\operatorname{Sin}(\theta o))}{35 \tilde{h}^{4}}-\frac{81 \tilde{Q}^{3} \alpha \tilde{\mu}^{3} \operatorname{Sec}(\theta o / 2)^{2}}{10 \tilde{h}^{7} \pi} \\
& -\frac{19683 \tilde{Q}^{5} \alpha^{2} \tilde{\mu}^{5} \operatorname{Sec}(\theta o / 2)^{2}}{200 \tilde{h}^{11} \pi}-\frac{6561 \tilde{Q}^{5} \alpha^{2} \tilde{\mu}^{5} \operatorname{Sec}(\theta o / 2)^{4}}{400 \tilde{h}^{11} \pi}=0=F(\tilde{Q})
\end{aligned}
$$

For the analysis, the numerical value of $\tilde{Q}$ can be obtained by Newton-Raphson formula

$$
\tilde{Q}_{i+1}=\tilde{Q}_{i}-\frac{F\left(\tilde{Q}_{i}\right)}{F^{\prime}\left(\tilde{Q}_{i}\right)}
$$

Taking appropriate initial value (eg. $\tilde{Q}=0$ ), accuracy of order $10^{-8}$ in the numerical value of $\tilde{Q}$ is obtained in two or three iterations. In the present analysis, convergence of order $10^{-12}$ is checked and selected by using Mathematica 7.0 which is sufficient accuracy for the analysis and discussion.

\section{FRICTIONAL TORQUE}

The frictional torque of the bearing shown in Fig. 1. is given by the following formula

$$
\tau=2 \pi \mu \int_{x_{o}}^{x_{R}} r(x)^{2} \frac{\partial^{2} v}{\partial z^{2}} d x
$$


It takes the dimensionless form

$$
\tilde{\tau}=2 \pi \tilde{\mu} \int_{\tilde{x}_{o}}^{1} \tilde{r}(\tilde{x})^{2} \frac{\partial^{2} \tilde{v}}{\partial \tilde{z}^{2}} d \tilde{x}
$$

where $\tilde{\tau}=\frac{\tau}{\mu_{N} R^{3} \omega}$.

\section{RESULTS AND DISCUSSIONS}

To study non-Newtonian and fluid inertia effects on the pressure and flow rate of externally pressurised flow between two curved surfaces of revolutions, numerical results for pressure and radial flow rate have been obtained for different values of parameter of pseudoplasticity, $\alpha\left(\alpha=\kappa P_{o}{ }^{2}\right)$ within the valid range of convergence of numerical solution procedure. Further, in order to prove the practical applicability of the problem, the value of pseudoplasticity parameter $\alpha$ is taken within $0 \leq \alpha \leq 1500$ based on the actual experimental results of (Wada \& Hayashi, 1971) for coefficient of pseudoplasticity $\kappa$ and viscosity $\mu$ and (Coombs and Dowson, 1964) for supply pressure $P_{o}$. The ranges of the coefficient $\mu$ and supply pressure $P_{o}$ are:

- coefficient of pseudoplasticity $(\kappa): 2 \cdot 10^{-6}-6 \cdot 10^{-6} \mathrm{~m}^{4} / \mathrm{N}^{2}$

- $\quad$ supply pressure $\left(P_{o}\right): 1.6 \cdot 10^{4} \mathrm{~N} / \mathrm{m}^{2}$

Further, in all the graphs, the values of pseudoplasticity coefficient $\kappa\left(\mathrm{m}^{4} / \mathrm{N}^{2}\right)$ have been shown in small braces with the values of pseudoplasticity parameter $\alpha$ to improve the applicability of the paper.

To obtain and analyse the numerical results for pressure distribution, and flow rate and to avoid the effect of cavitation, the value of speed parameter $\mathrm{S}$ is selected in the range $0 \leq \mathrm{S} \leq 0.5$ for plane parallel plates and $0 \leq \mathrm{S}<1$ for spherical case.

\subsection{Case of plane parallel plates}

Fig. 4. and Fig. 5. show the variation of dimensionless pressure distribution with respect to the radius of the bearing for different values of fluid parameter $\alpha$ (hence with $\kappa$ ) and rotation parameter $S$. The pressure for $\alpha=0$ (i.e. $\kappa=0$ ) obtained by the present methodology agrees with the Dowson's experimental and theoretical results up to a sufficient degree of accuracy due to which the pressure curves for $(\alpha=0)$ overlap the Dowson's experimental and theoretical results (Coombs and Dowson, 1964). It is observed that the pressure for pseudoplastic fluids $(\alpha>0)$ is higher than that with Newtonian fluids $(\alpha=0)$ at each value of $\tilde{r}$ and every value of rotation parameter $S$.

Moreover, the pressure for pseudoplastic fluids increases with the increase of the fluid parameter $\alpha$. Since, the supply pressure $\left(P_{o}\right)$ is constant, the pressure for pseudoplastic fluids increases with the increase of the rheological parameter $\kappa$. However, the difference in the pressure distribution with the Newtonian and pseudoplastic fluids is small in the case of no rotation and the difference increases as the rotation increases. The variation of pressure from Newtonian to pseudoplastic fluids is also dependent on the film thickness. The larger the film thickness, the higher is the variation of pressure due to pseudoplasticity. As the bearings are merely operated at a high speed in practical situations, the effect of pseudoplastic fluids dominantly increases the pressure distribution - a result in agreement with (Hung, 2009; Lin, 2001; Usha and Vimla, 2000; Wada and Hayashi, 1971) and consistent with the real situation of the problem. 


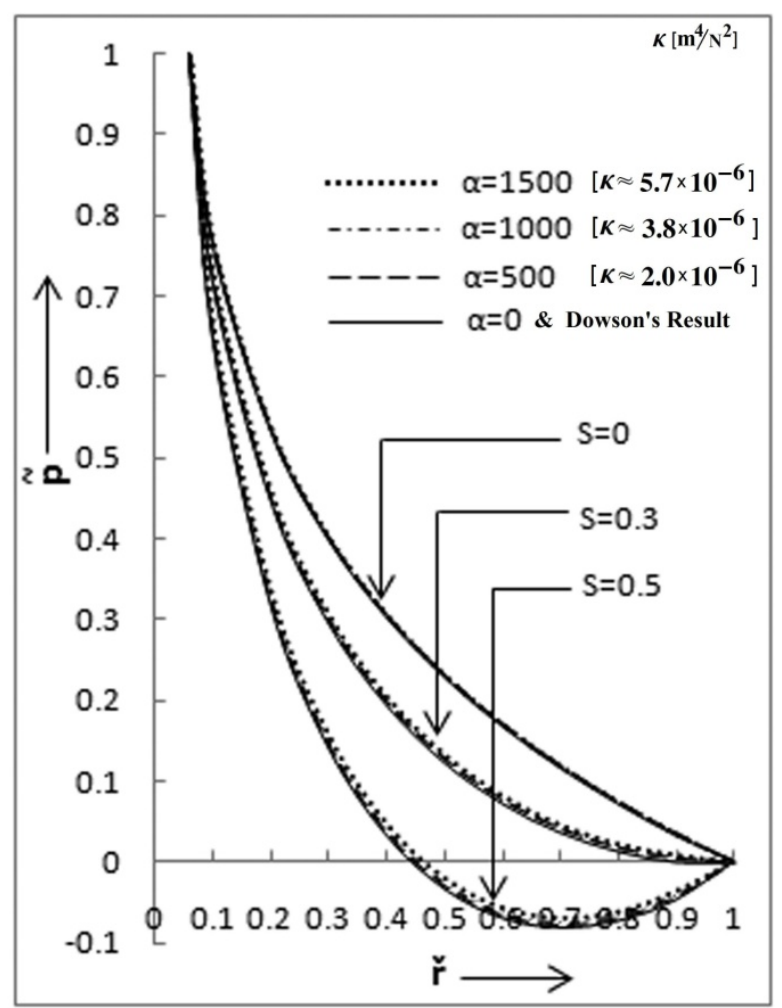

Fig. 4. Variation of pressure $\tilde{p}$ in the clearance between parallel plates with radius of the plate $\tilde{r}$ for different values of fluid constant $\alpha$ and rotation parameter S. $\tilde{h}=0.001$

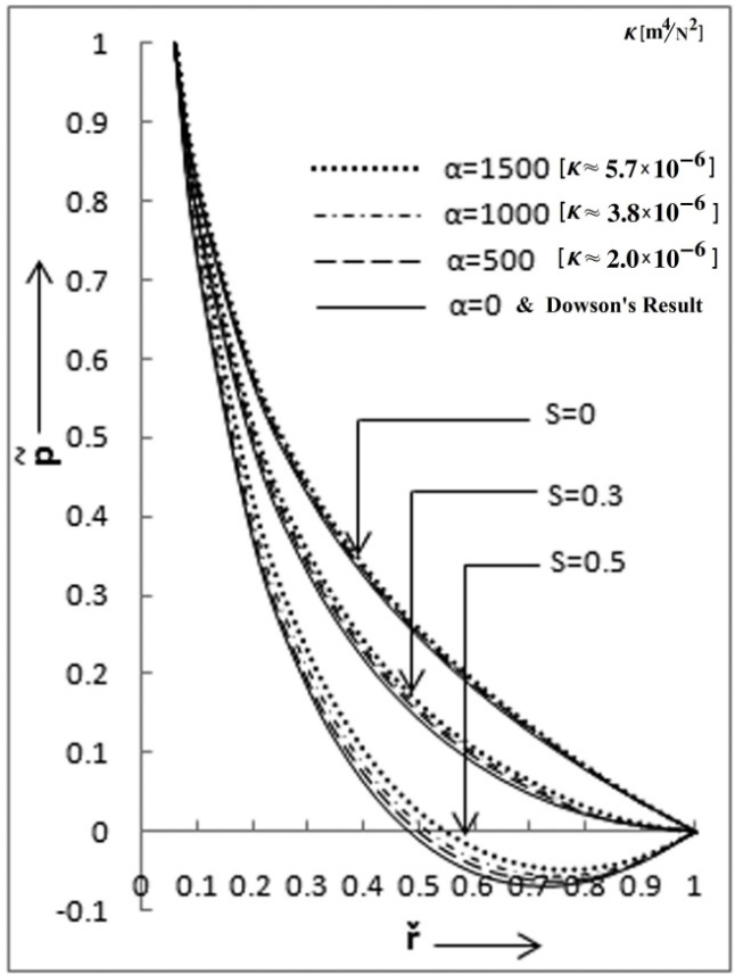

Fig. 5. Variation of pressure $\tilde{p}$ in the clearance between parallel plates with radius of the plate $\tilde{r}$ for different values of fluid constant $\alpha$ and rotation parameter S. $\tilde{h}=0.003$ 
Fig. 6. shows the variation of dimensionless frictional torque of the bearing with respect to the normalised viscosity $\tilde{\mu}=\mu / \mu_{N}$ and pseudoplasticity coefficient $\kappa$ for different values of the dimensionless film thickness. It is observed that the frictional torque increases with the decrease of pseudoplasticity coefficient $\kappa$. Since the viscosity of the fluid increases and pseudoplasticity coefficient decreases with the amount of the additive (viscosity index improver), it is therefore concluded that the frictional torque increases with the increase of viscosity. The rate of variation of the dimensionless frictional torque also decreases with the increase of film thickness.

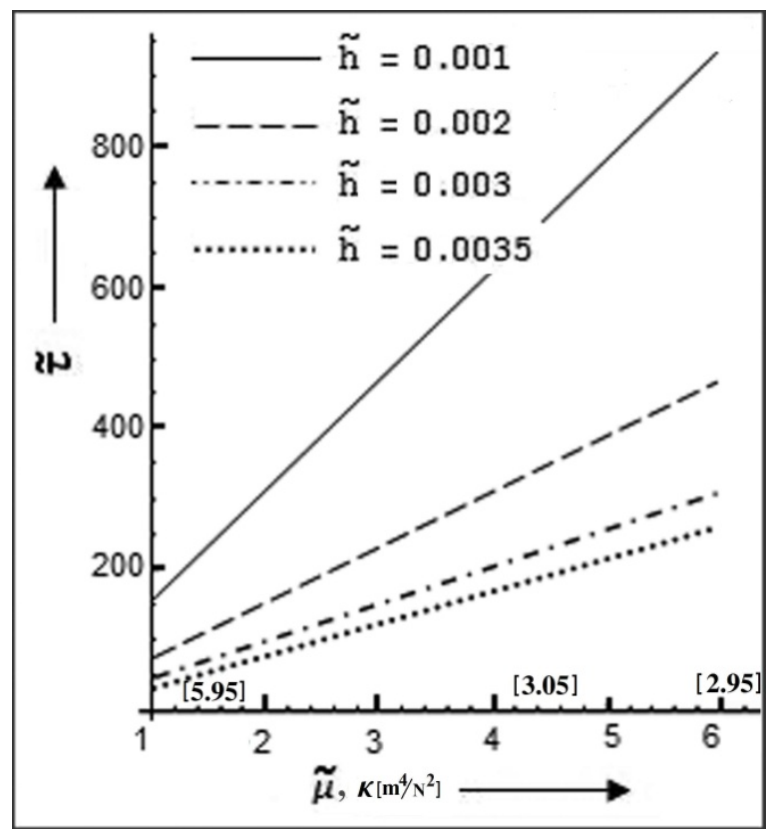

Fig. 6. Variation of dimensionless frictional torque $\tilde{\tau}$ with normalised viscosity $\tilde{\mu}$ in the case of plane parallel plates for different values of film thickness $\tilde{h}$

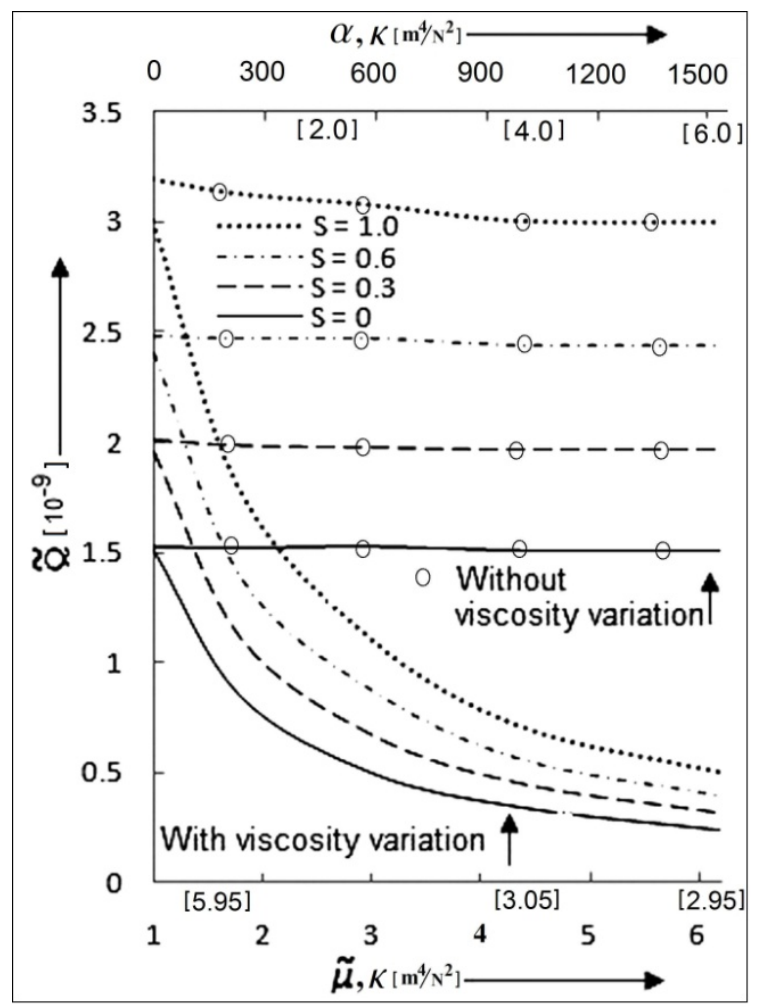

Fig. 7. Variation of dimensionless radial flow rate $\tilde{Q}$ in the clearance between parallel plates with normalised viscosity $\tilde{\mu}$ and the fluid constant $\alpha$ for different values of rotation parameter $\mathrm{S}$ 
Fig. 7. shows the variation of dimensionless flow rate with respect to the normalised viscosity $\tilde{\mu}=\mu / \mu_{N}$ and fluid parameter $\alpha$ (and corresponding $\kappa$ ) for different values of rotation parameter $S=0,0.3,0.6,1.0$. The flow curves with small circles show the variation of flow rate with respect to fluid parameter $\alpha$ (i.e. with $\kappa$ ) when the fluid viscosity is constant and the curves without circles show the variation of flow rate with respect to normalised viscosity $\tilde{\mu}$. It is observed that the flow rate decreases with the increase of viscosity for each value of rotation parameter $\mathrm{S}$ but it remains almost same with respect to fluid parameter $\alpha$ (i.e. with $\kappa$ ) if the viscosity remains constant. Thus, if the viscosity of the fluid does not vary, the flow rate has negligible variation with the pseudoplasticity of the fluid.

\subsection{Case of concentric spheres}

Fig. 8. and Fig. 9. show the variation of dimensionless pressure distribution with respect to the bearing angle $\theta$ (as shown in Fig. 3) for different values of fluid parameter $\alpha$ (hence with $\kappa$ ) and rotation parameter $S$. It is observed that the pressure in the case of pseudoplastic fluids $(\alpha>0)$ is higher than that in the case of Newtonian fluids $(\alpha=0)$ at each value of $\theta$ and every value of rotation parameter $S$. The pressure for pseudoplastic fluids increases with the increase of the fluid parameter $\alpha$ (i.e. with $\kappa$ ). Further, the difference in the pressure distribution with the Newtonian and pseudoplastic fluids is small in the case of no rotation and the difference increases with the increase of rotation parameter $S$. At a speed $(S=0.95)$, the effect of pseudoplastic fluids significantly increases the pressure distribution which can be clearly seen from Fig. 9. However, with small film thickness $\tilde{h}=0.001$, the effect of pseudoplasticity on pressure distribution is observed to be small in comparison to the case of an average film thickness $\tilde{h}=0.003$. It shows that the effect of pseudoplasticity is also dependent on the film thickness which has been already discussed in the case of plane plates. It is also clear from the Figures 4, 5, $8 \& 9$ of pressure distribution that the effect of pseudoplasticity is more significant in the spherical case than the plane plates with the similar parameters and also, the results agree in nature with (Hung, 2009; Lin, 2001; Usha and Vimla, 2000; Wada and Hayashi, 1971).

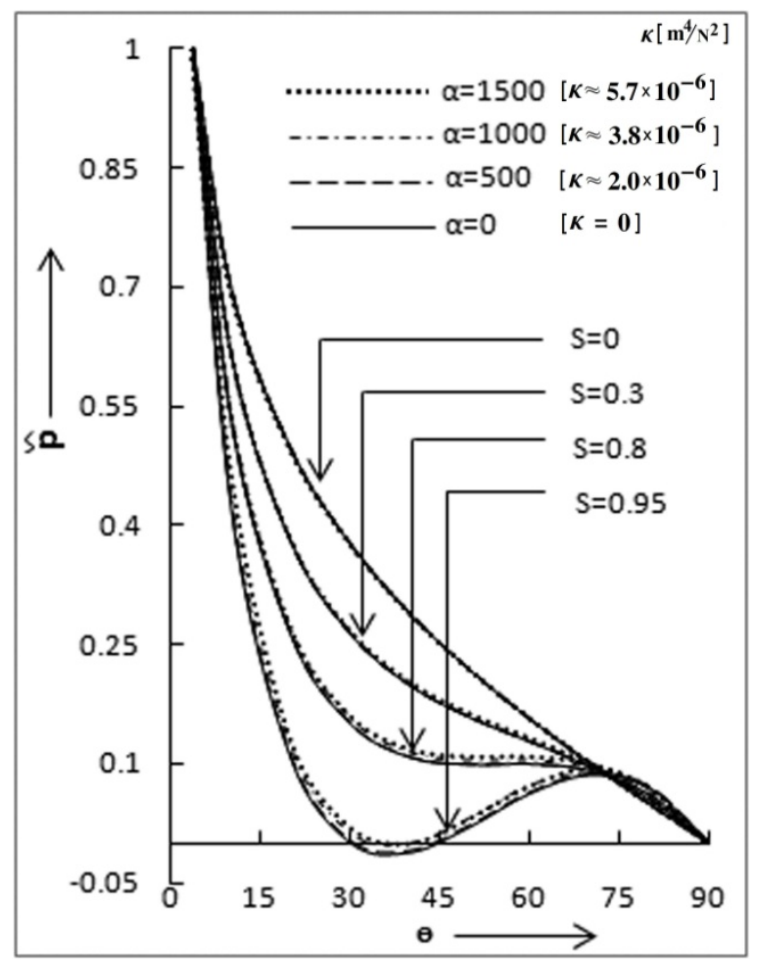

Fig. 8. Variation of film pressure $\tilde{p}$ in narrow clearance $(\tilde{h}=0.001)$ between concentric spherical surfaces with angle $\theta$ for different values of fluid constant $\alpha$ and rotation parameter $S$ 


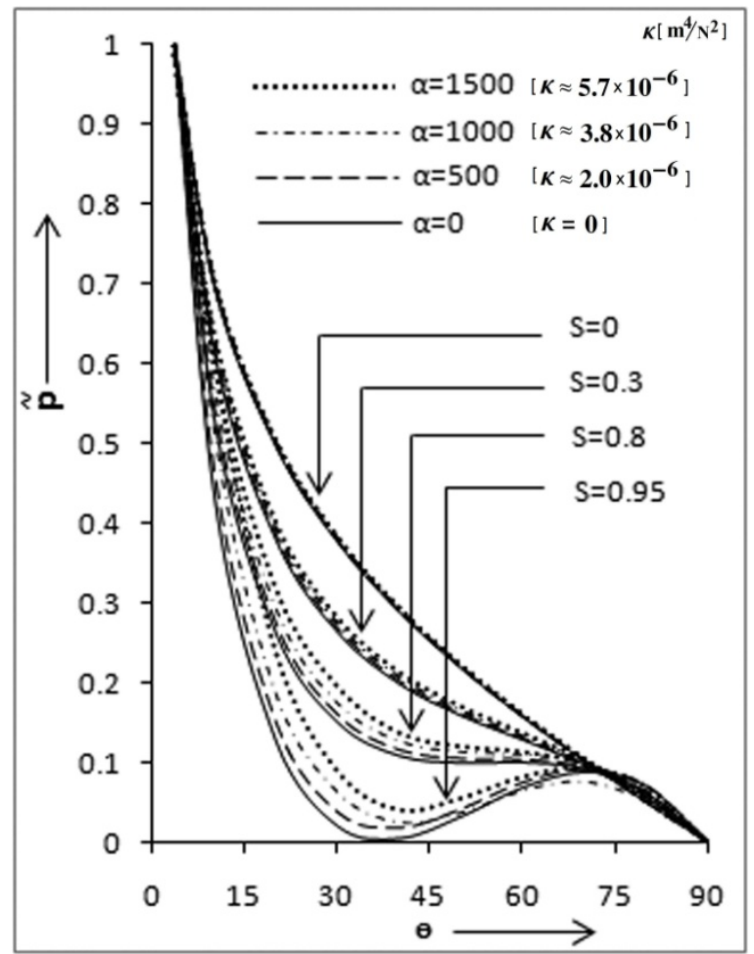

Fig. 9. Variation of film pressure $\tilde{p}$ in average clearance $(\tilde{h}=0.003)$ between concentric spherical surfaces with angle $\theta$ for different values of fluid constant $\alpha$ and rotation parameter $S$

Fig. 10. shows the variation of dimensionless frictional torque generated due to the rotation of the bearing with respect to the normalised viscosity $\tilde{\mu}=\mu / \mu_{N} \& \kappa$ for different values of the dimensionless film thickness. The variation of the frictional torque with the viscosity is similar as in the case of plane plates i.e. the dimensionless frictional torque increases with the increase of normalised viscosity and hence it decreases with the increase of pseudoplasticity coefficient $\kappa$. The frictional torque also decreases with the increase in film thickness.

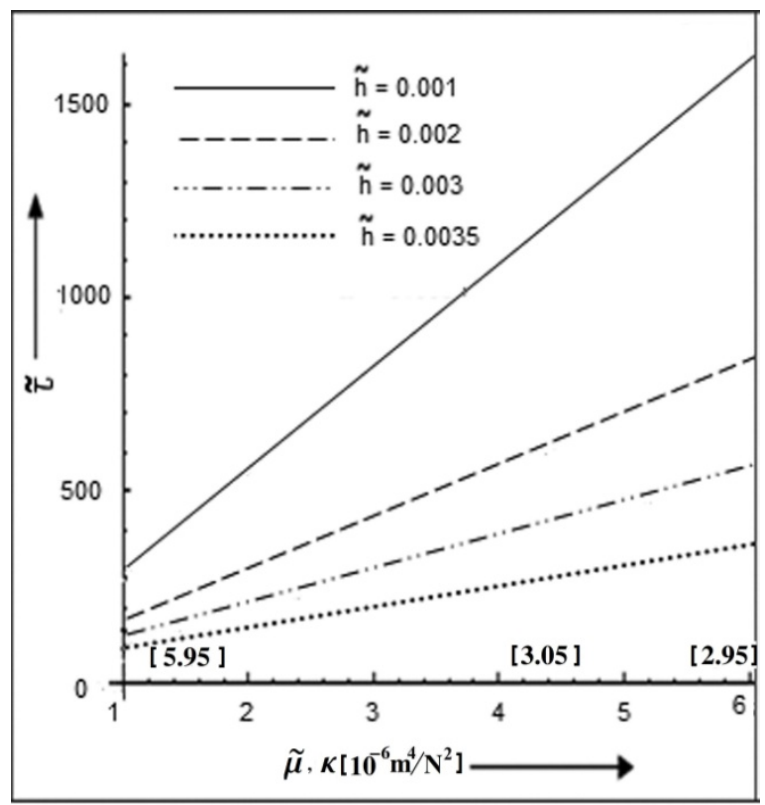

Fig. 10. Variation of dimensionless frictional torque $\tilde{\tau}$ with normalised viscosity $\tilde{\mu}$ in the case of concentric spherical surfaces for different values of film thickness $\tilde{h}$ 
Fig. 11. shows the variation of dimensionless flow rate with respect to the normalised viscosity $\tilde{\mu}=\mu / \mu_{N}$ and fluid parameter $\alpha$ (i.e. with $\kappa$ ) for different values of rotation parameter $S=0,0.3,0.6,1$. The flow curves with small circles show the variation of flow rate with respect to fluid parameter $\alpha$ (and hence with $\kappa$ ) when the fluid viscosity is constant and the curves without circles show the variation of flow rate with respect to normalised viscosity $\tilde{\mu}$. The variation of flow rate in this case is also similar as in the case of plane parallel plates i.e. the flow rate decreases with the increase of viscosity for each value of rotation parameter $S$ but it shows a negligible variation with respect to fluid parameter $\alpha$ (and hence with $\kappa$ ) for constant viscosity.

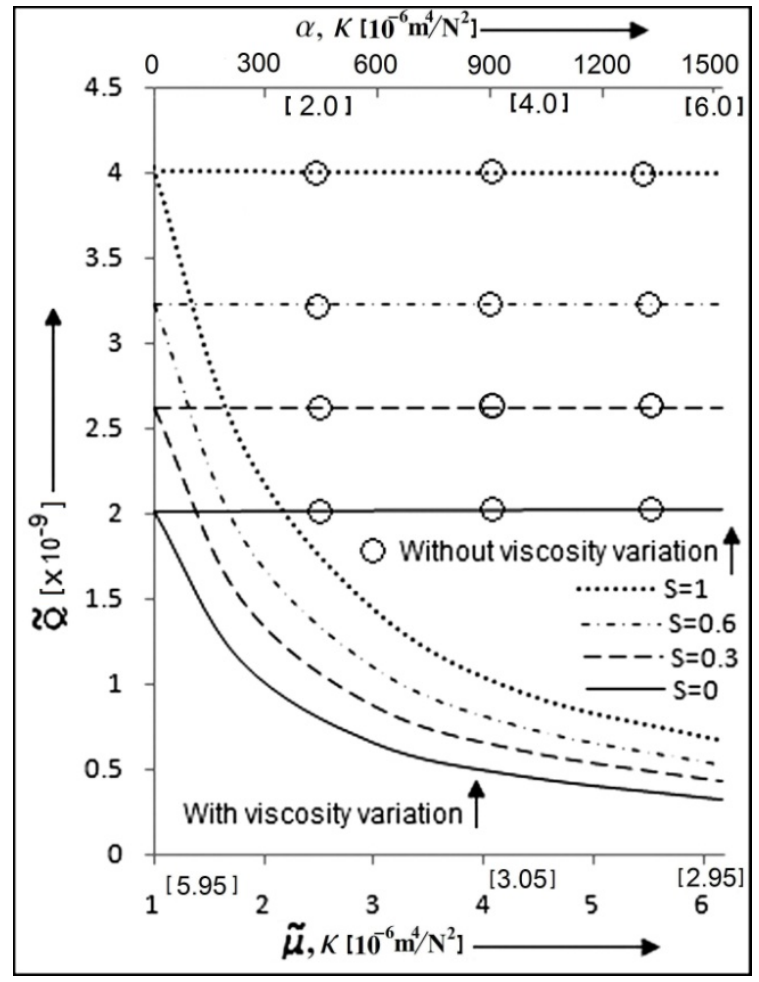

Fig. 11. Variation of fluid flow rate $\tilde{Q}$ in clearance between concentric spherical surfaces with viscosity variation $\tilde{\mu}$ and the fluid constant $\alpha$ for different values of rotation parameter $S$

From the discussions of Figures 4-11, it is clear that the effect of pseudoplasticity is attributable to the pressure distribution due to its nature of stress strain relationship. But, the effect of pseudoplasticity on the flow rate and frictional torque is due to the change of viscosity.

It is further concluded that the effect of pseudoplasticity on pressure distribution is small in the case of no rotation $(S=0)$, but in the case of large values of $S$ (i.e. high rotation or large $R$ ) the fluid inertia becomes significant and in this case the deviation of pressure distribution for pseudoplastic lubricant can be easily observed in both the cases of plane parallel plates and the concentric spherical surfaces.

\section{CONCLUSIONS}

The pseudoplastic effects of an isothermal incompressible non-Newtonian lubricant on the pressure distribution, frictional torque and flow rate of curvilinear bearings, neglecting the radial fluid inertia and cavitation effects, are presented with examples of plane parallel plates and concentric spherical surfaces. 
The Rabinowitsch fluid i.e. cubic equation model is considered to derive the analytic expressions of pressure using the Energy Integral Method. The following conclusions are drawn:

- The method is simpler and comprehensive because the formation of modified Reynolds equation can be avoided.

- Comparing with the Newtonian case, the dimensionless film pressure distribution with the pseudoplastic lubricants is higher. Further, with the increase of the rheological parameter $\kappa$, there is an increase in the film pressure in both cases of the plane parallel plates and concentric spherical surfaces.

- The effect of pseudoplasticity on the pressure distribution is small in the case of no rotation and increases with the rotation due to inertia effect.

- The effect of pseudoplasticity is comparatively significant for pressure distribution with large radius $R$ due to high inertia effect.

- The flow rate of pseudoplastic fluids decreases due to increase of viscosity i.e. the effect of viscosity index improver - viscosity thickener decreases the flow rate.

- The frictional torque of pseudoplastic fluids increases due to increase in viscosity i.e. the effect of viscosity index improver - viscosity thickener increases the frictional torque and hence the frictional torque in pseudoplastic case is always higher than in the Newtonian case.

Hence, the variation of pressure distribution is dependent on the cubic stress nature of pseudoplastic fluids and the variations of the flow rate and the frictional torque are viscosity dependent. All the variations are consistent with the physical nature of the problem.

\section{SYMBOLS}

$\begin{array}{ll}h & \text { film thickness, } \mathrm{m} \\ \tilde{h} & \frac{h}{x_{R}},- \\ p & \text { film pressure, } \mathrm{N} / \mathrm{m}^{2} \\ \tilde{p} & \frac{p}{P_{o}},- \\ P_{o} & \text { supply pressure, } \mathrm{N} / \mathrm{m}^{2} \\ Q & \text { fluid flow rate, } \mathrm{m}^{3} / \mathrm{s} \\ \tilde{Q} & \frac{\mu_{N}}{\pi R x_{R}^{2} p_{O}} Q,- \\ R & \text { maximum outer radius of the surfaces, } \mathrm{m} \\ \tilde{r} & \frac{r}{R},- \\ \tilde{r}_{o} & \frac{r_{o}}{R},- \\ S & \frac{3}{20} \frac{\rho R^{2} \omega^{2}}{P_{o}},- \\ \tilde{u} & \frac{u}{R \omega},- \\ \tilde{v} & \frac{v}{R \omega},- \\ x_{o}, r_{o}, \theta_{o} & \text { radius of supply hole, } \mathrm{m}\end{array}$


$\tilde{z} \quad \frac{z}{x_{R}},-$

Greek symbols

$\begin{array}{ll}\alpha & \kappa P_{o}^{2},- \\ \kappa & \text { coefficient of pseudoplasticity, } \mathrm{m}^{4} / \mathrm{N}^{2} \\ \mu & \text { viscosity of the fluid, } \mathrm{kg} /(\mathrm{m} \cdot \mathrm{s}) \\ \mu_{N} & \text { viscosity of Newtonian fluid, } \mathrm{kg} /(\mathrm{m} \cdot \mathrm{s}) \\ \tilde{\mu} & \frac{\tilde{\mu}}{\mu_{N}},- \\ \rho & \text { density of fluid, } \mathrm{kg} / \mathrm{m}^{3} \\ \tau_{x z} & \text { shearing stress, } \mathrm{N} / \mathrm{m}^{2} \\ \tau & \text { frictional torque, } \mathrm{N} \cdot \mathrm{m} \\ \tilde{\tau} & \frac{\tau}{2 \pi \mu_{N} R^{3} \omega},- \\ & \frac{\tau_{x z}}{P_{o}},- \\ \tau_{x z} & \text { angular velocity of runner, } \mathrm{rad} / \mathrm{s} \\ \omega & \end{array}$

\section{REFERENCES}

Bourging P., Gay B., 1984. Determination of the load capacity of finite width journal bearing by finite element method in the case of a non-newtonian lubricant. ASME J. Tribol., 106, 285-290. DOI:10.1115/1.3260906.

Cameron A., 1996. Basic Lubrication Theory, Ellis Harwood, Chichester, 1996.

Coombs J. A., Dowson D., 1964. An experimental investigation of the effects of lubricant inertia in a hydrostatic thrust bearing. Proc. Inst. Mech. Engrs., London, 179 (Paper 12), 96-108. DOI:10.1243/PIME_CONF_1964_179_270_02.

Cross M.M., 1965. Rheology of non-Newtonian fluids: a new flow equation for pseudoplastic systems. J. Colloid Sci., 20, 417-437. DOI:10.1016/0095-8522(65)90022-X.

Elkouh A. F., 1967. Inertia effect in laminar radial flow between parallel plates. Int. J. Mech. Sci., Pergamon Press Ltd., 9, 253-255. DOI:10.1016/0020-7403(67)90020-3.

Giannikos C., Buckholz R. H., 1988. Elastic bearings lubricated with non-Newtonian power law fluids - a boundary element approach. Tribology Trans., 31, 105-112. DOI:10.1080/10402008808981805.

Hanks R. W., 1979. The axial flow of yield-pseudoplastic fluids in a concentric annulus. Ind. Eng. Chem. Process Des. Dev., 18, 488-493. DOI: 10.1021/i260071a024.

Hashimoto H., Wada S., 1986. The effects of fluid inertia forces in parallel circular squeeze film bearings lubricated with pseudoplastic fluids. ASME J. Tribol., 108, 282-287. DOI:10.1115/1.3261177.

Hsu Y. C., Saibel E., 1965. Slider bearing performance with a non-newtonian lubricant. ASLE Trans., 8, 191-194.

Hung C. R., 2009. Effects of non-newtonian cubic-stress flow on the characteristics of squeeze film between parallel plates. Education Specialization in 97P-009, 97, 87-97 (洪啟仁, 非牛頓三次方應力流變效應對於平行板系統擠壓性能影響之研究, 教專研 97P-009, 87-97).

Jurczak P., Walicka A., Walicki E., Michalski D., 2006. Influence of rheological parameters on the mechanical parameters of curvilinear thrust bearing with one porous wall lubricated by a couple stress fluid. Int. J. Appl. Mech. Eng., 11, 221-233.

Kapur V. K., Verma K., 1973. Energy integral approach for hydrostatic thrust bearing. Japanese J. App. Phy., 12, 1070. DOI: 10.1143/JJAP.12.1070.

Khonsari M. M., Brewe D. E., 1989. On the performance of finite journal bearings lubricated with micropolar fluids. Tribology Trans., 32, 155-160.

Lin J. R., 1999. Static and dynamic characteristics of externally pressurized circular step thrust bearings lubricated with couple stress fluids. Tribology Int., 32, 207-216. DOI:10.1016/S0301-679X(99)00034-1. 
Lin J. R., 2001. Non-newtonian effects on the dynamic characteristics of one dimensional slider bearings : rabinowitsch model. Tribology Letters, 10, 237-243. DOI: 10.1023/A:1016678208150.

Pinkus O., Sternlicht B., 1961. Theory of hydrodynamic lubrication. McGra-Hill Book Company, Inc, New York. Savins J.G., 1958. Generalised Newtonian (pseudoplastic) flow in stationary pipes and annuli. Trans. AIME, 213, 325-332.

Serangi M., Majumda B. C., Sekhar A. S., 2005. Elastohydrodynamically lubricated ball bearings with couple stress fluids, part 1: steady state analysis. Tribology Trans., 48, 404-414.

Shukla J. B., Prasad K. R., Chnadra P., 1982. Effects of consistency variation of power law lubricants in squeeze films. Wear, 76, 299 - 319. DOI:10.1016/0043-1648(82)90069-2.

Usha R., Vimla P., 2000. Fluid inertia effects in a non-newtonian squeeze film between two plane annuli. Trans. ASME, 122, 872-875. DOI:10.1115/1.1288928.

Wada S., Hayashi H., 1971. Hydrodynamic lubrication of journal bearings by pseudoplastic lubricants. Bulletin of JSME, 14 (No. 69), 279-286.

Walicka A., Falicki J., 2010. Pressurized flow of the Herschel-Bulkley fluid in a clearance between fixed surfaces of revolution. Chem. Process Eng., 31, 199-215.

Walicka A., Falicki J., 2010. Inertia effects in the flow of a simple Casson fluid between two fixed surfaces of revolution. Chem. Process Eng., 30, 603-619. 